\title{
Bio-degradable material realization for antenna fabrication in wireless communications
}

\author{
Thanakorn Homsai ${ }^{1}$, Chuwong Phongcharoenpanich ${ }^{2}$, and Wipoo Sriseubsai ${ }^{*}$ \\ ${ }^{1}$ Industrial Engineering Department, Faculty of Engineering, King Mongkut's Institute of Technology Ladkrabang, Bangkok, Thailand \\ ${ }^{2}$ Telecommunication Engineering Department, Faculty of Engineering, King Mongkut's Institute of Technology Ladkrabang, \\ Bangkok, Thailand
}

\begin{abstract}
The bio-degradable PBS material is presented to be applied for microwave device design (antenna). The PBS is forming by using compression molding. The PBS thickness is $1 \mathrm{~mm}$ with the flexible characteristic. In this paper, the antenna design for the operating frequency band of $2400-2500 \mathrm{MHz}$ is design using the bio-degradable material with the dielectric constant of 3.2. The prototype was fabricated. The characteristics of the proposed device were presented. The effected of the curved antenna and substrate are described in this work.
\end{abstract}

\section{Introduction}

The assumption of the technology product has been rapidly grown because nowadays the technology become a part of human daily life. Eevery day many electronic and microwave devices are improved in both of the model and characteristics to support the modern and advance technology [1]. This creates the plenty of electronic waste (e-waste) which is included the e-waste from the end-of-life electronic equipment. Therefore, the e-wastes become a considerable problem and needs immediately reaction nowadays [2]-[3]. Many techniques are presented to decrease e-wastes such as recycling, using environmentally friendly electronics [4]

No one can be denied that one of an important technology is a wireless communication technology because it can work efficient without wire-connection having more flexibility, infrastructure cost saving, improve customer service, enhanced collaboration and others. To communication by wireless technology, three basically components namely transmitter, receiver and medium are need. Mostly common wireless communication uses radio wave; therefore, an antenna is important to convert transmitted electrical signal to electromagnetic wave (EM wave) propagated in directing direction [5]. At the receiver, the antenna coverts the EM wave to electrical signal.

As same as modern technology, the changing of trend of technology and model of wireless device, the changing of antenna and printed circuit board is relatively important. Normally, the antenna for modern wireless communication are located on a printed circuit board as a substrate of the antenna. The substrate properties are effect to size and characteristics of the antenna. For the antenna design, the antenna substrate can improve or decrease electrical and mechanical stability of a device. There are a lot of commercial substrate such as epoxy, FR-4, PET, PE and many others. However, they cannot decay or hardly degradable by nature. Hence, the bio-degradable substrate is one of the way to decrease e-waste. Using biological materials and products are a possibility for decreasing amounts and recycling of waste.

The bio-gradable material is a material that can be broken down by natural process and it can be returned to environment or reused [6]. The bio-polymer becomes an interesting choice to be the antenna substrate. In this paper, the polybutylene succinate (PBS) which is a biodegradable material is provided for microwave device (antenna). The PBS has a highly flexible, heat resistant, easy to compression. PBS is used in a large scale of production such as eco-smart food packaging, agriculture, pharmaceutical, automotive part, electronic structure part and others [7].

The applied of PBS for antenna design is studied and designed to ensure that the presented bio-degradable based device is proper for wireless communication technology. The compression process and the characteristics of the proposed device such as impedance, reflected power, radiation pattern and gain are described in this work. An introduction provides the detail about the basic theory, importance, and the detail of this work. In section II, the compression process is expressed. The design of the device shows in section III.

\footnotetext{
* Corresponding author: wipoo.sr@kmitl.ac.th
} 
The characteristics of the bio-martial based device are presented in section IV. Final section provides the conclusion of this work.

\section{PBS compression process}

For preparation process of PBS forming, the devices consist of 1. Polybutylene succinate (FZ91PM) 2. LABcompanion oven 3 . Weighing scales 4 . Mold with the size of $18 \mathrm{~cm} \times 18 \mathrm{~cm}$ with the thickness of $1 \mathrm{~mm}$. To determine the volume for PBS forming, the volume can be calculated from $D=m / v$. Where, $m=$ Mass (Gram), $D$ $=$ density of polybutylene succinate (Gram/ cubic centimetre) and $v=$ volume (cubic centimetres). The density of polybutylene succinate is $1.26 \mathrm{ram} /$ Cubic centimetre. The forming process is described in figure 1.

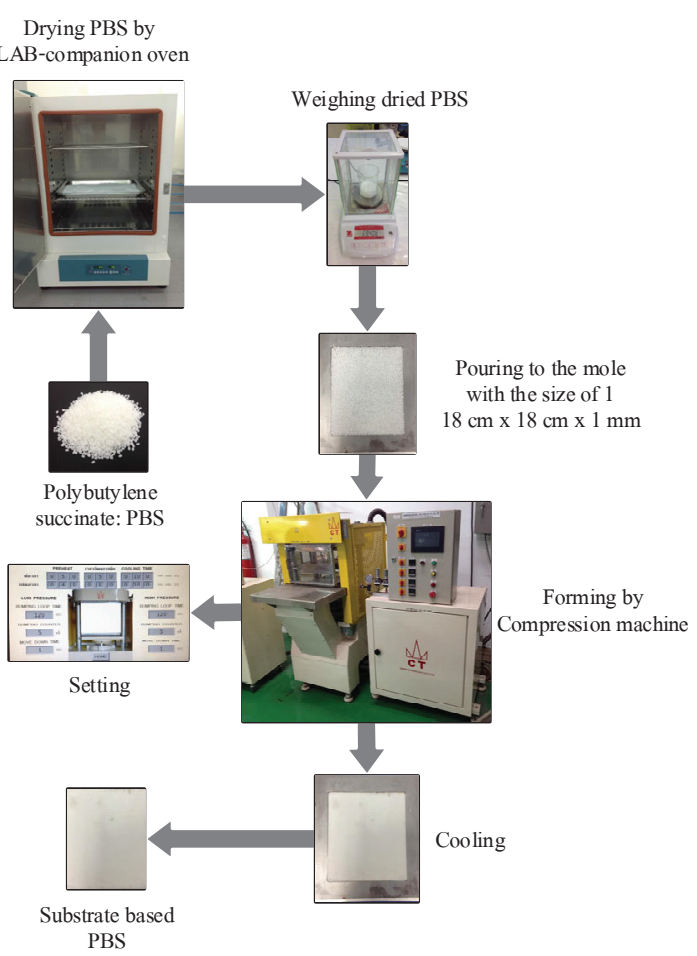

Fig. 1. Forming process of PBS substrate

One of the important process is drying process. The PBS (FZ91PM) is dried by heating at 80 degrees with 8 hours to decrease humidity in the material. The humidity of material causes air bubble making nonhomogeneous in a texture of material. The nonhomogeneous can decrease antenna efficiency. The dried PBS is weighed to find the mass for a compression process. The dielectric constant is measured by $85070 \mathrm{E}$ Dielectric Probe Kit together with Network Analyzer. The measured dielectric of fabricated PBS is 3.2.

\section{Apply of material on antenna design}

The PBS is applied to be an antenna substrate with the thickness of $1 \mathrm{~mm}$. The radiated patch used a stick copper patch. The designed antenna is based on a printed circular monopole fed by coplanar waveguide. A size of the circular monopole is related with the wavelength of the operating frequency. The wavelength $(\lambda)$ is the ratio of frequency $(f)$ divided by speed of light $(C)$. The initial printed monopole size can be calculated via $L=\left(L_{d}+2 r\right)$. Where $L_{d}=$ length of monopole, $r=$ radius of monopole and $L=$ length of feeding monopole. The size of an initial parameter (structure A) tabulated in table I and the parameter of developed structure (structure B) is tabulated in table II. Table III shows the parameter of the proposed antenna. From the initial stature, the parameter is adjusted to achieve the desired characteristics. The improvement of reflection power from each structure is provided in figure 2 . The structure and the photograph of the proposed antenna are shown in figure 3 .

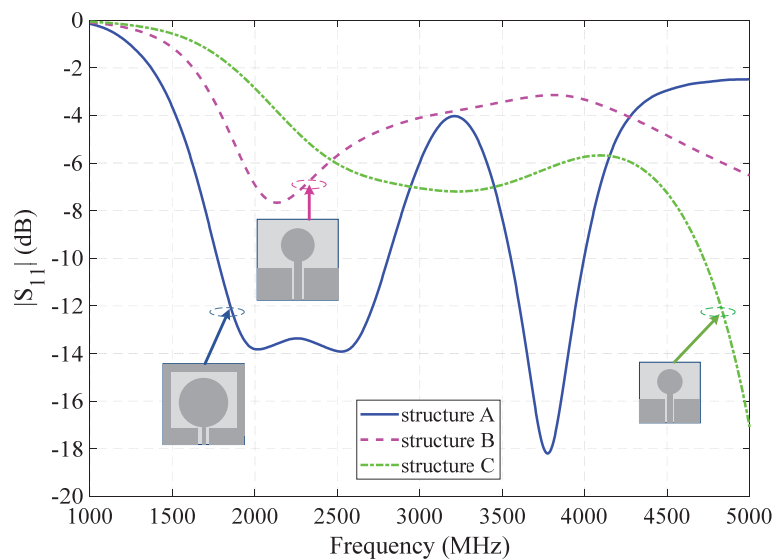

Fig. 2. The developed structure of the PBS-based antenna

Table 1. Initial antenna parameters

\begin{tabular}{cc|ll}
\hline Parameters & $\begin{array}{l}\text { Physical size } \\
(\mathrm{mm})\end{array}$ & Parameters & $\begin{array}{l}\text { Physical size } \\
(\mathrm{mm})\end{array}$ \\
\hline$L$ & 37 & $W_{f}$ & 3.23 \\
\hline$W$ & 37 & $S$ & 1.00 \\
\hline$H$ & 1 & $L_{d}$ & 3.00 \\
\hline$L_{g}$ & 15 & $r$ & 8.00 \\
\hline
\end{tabular}

Table 2. Developed antenna parameters

\begin{tabular}{cc|ll}
\hline Parameters & $\begin{array}{l}\text { Physical size } \\
(\mathrm{mm})\end{array}$ & Parameters & $\begin{array}{l}\text { Physical size } \\
(\mathrm{mm})\end{array}$ \\
\hline$L$ & 50 & $W_{f}$ & 3.23 \\
\hline$W$ & 50 & $S$ & 1.00 \\
\hline$H$ & 1 & $L_{d}$ & 0.58 \\
\hline$L_{g}$ & 11 & $r$ & 15.5 \\
\hline
\end{tabular}


The initial parameters are calculated based on printed circular monopole fed by coplanar waveguide. Owing to integrating of antenna with a SMA connector, the characteristic of the antenna changes as shown in figure 2. It is obvious that the antenna resonates at the relatively high frequency. Then, the parameters are developed by changing the antenna size. From improving of the antenna size, the trend of the operating frequency is shifted to the lower frequency. However, the reflected power is quite higher than that of desired reflection power (less than $-10 \mathrm{~dB}$ ). Therefore, to meet the requirement, the antenna structure is revised. The proposed antenna that meet the requirement is presented in figure 4.

Table 3. Antenna parameters

\begin{tabular}{lc|ll}
\hline Parameters & $\begin{array}{l}\text { Physical size } \\
(\mathrm{mm})\end{array}$ & Parameters & $\begin{array}{l}\text { Physical size } \\
(\mathrm{mm})\end{array}$ \\
\hline$L$ & 50 & $W_{f}$ & 2.00 \\
\hline$W$ & 50 & $S$ & 1.00 \\
\hline$H$ & 1 & $L_{d}$ & 0.51 \\
\hline$L_{g}$ & 11 & $r$ & 15.5 \\
\hline$W_{m}$ & 3.5 & $L_{m}$ & 4.00 \\
\hline
\end{tabular}

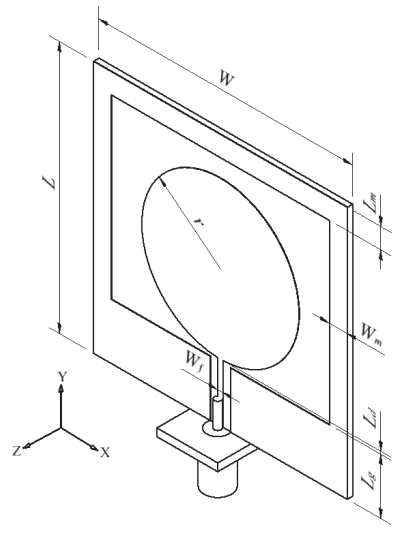

(a) Structure

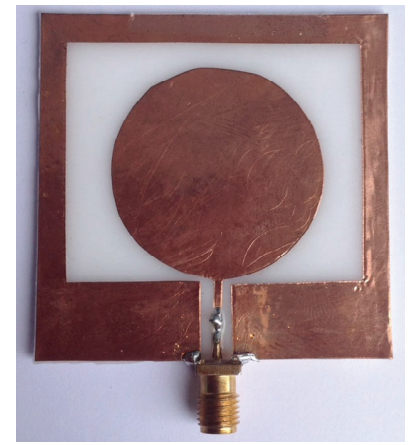

(b) Photograph

\section{Characteristic of the Proposed Device}

To achieve a maximum power transfers, the antenna and transmission line should be matched impedance. A normal antenna always designs to match with $50 \mathrm{ohms}$ of transmission line system along the proposed operating frequency. Hence, the antenna should have the impedance close to 50 ohms. In figure 4, it is illustrated that the antenna impedance is average nearly $50 \mathrm{ohms}$ having around $49.2 \mathrm{ohms}$ from the operating frequency between 2400 to $2500 \mathrm{MHz}$.

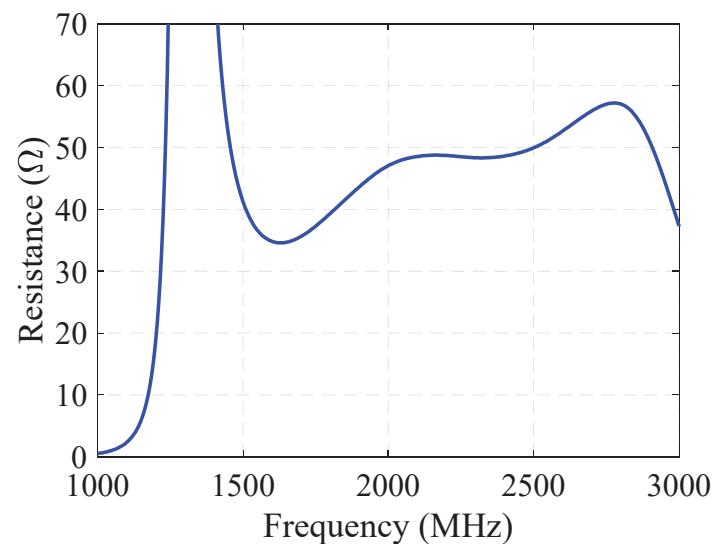

(a) Resistance

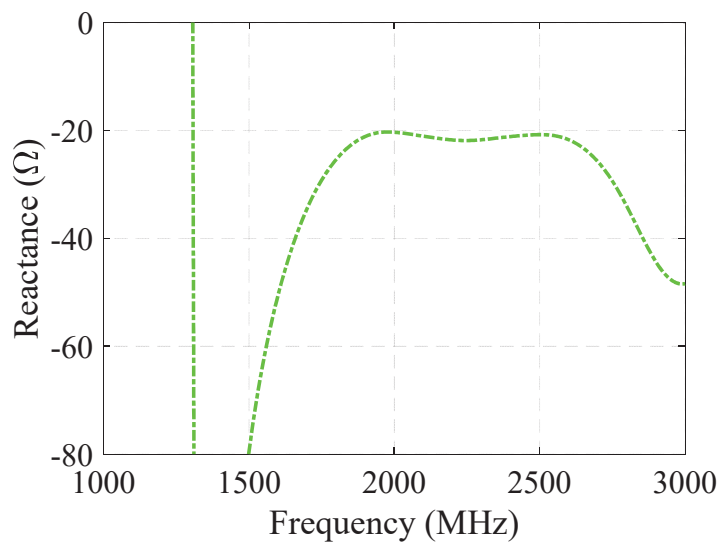

(b) Reactance

Fig. 4. Impedance of the proposed antenna

The impedance bandwidth is defined as the range of the operating frequency that the reflection power is acceptable with the transmission line. The reflected power should have a less value normally should be less than $-10 \mathrm{~dB}$ for the desired operating frequency resulting in the limitation of operating frequency of the designed antenna. In this work, impedance bandwidth is 1020 $\mathrm{MHz}$ (1780 to $2800 \mathrm{MHz}$ ) covering the proposed operating frequency (2400 to $2500 \mathrm{MHz}$ ).

Fig. 3. The PBS-based antenna 


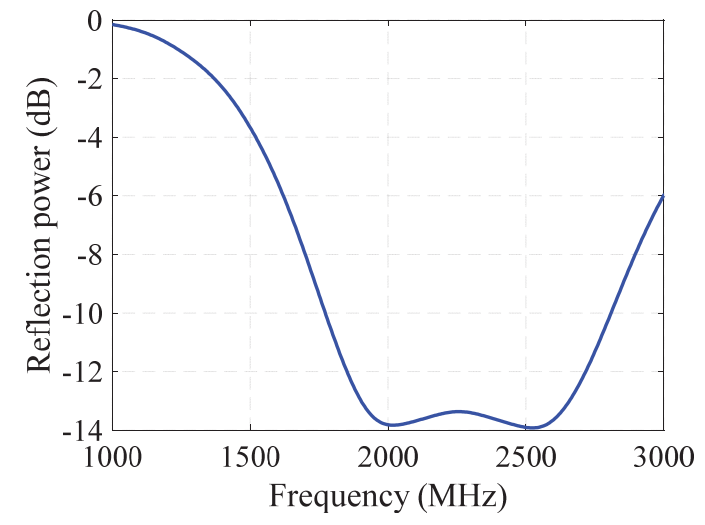

Fig. 5. Reflection power of the proposed antenna

The antenna gain is one of an important parameter that effect to the communication range. Gain of the antenna is normally describes in decibel $(\mathrm{dB})$. The proposed antenna gain is $3.12 \mathrm{dBi}$ as presented in figure 6.

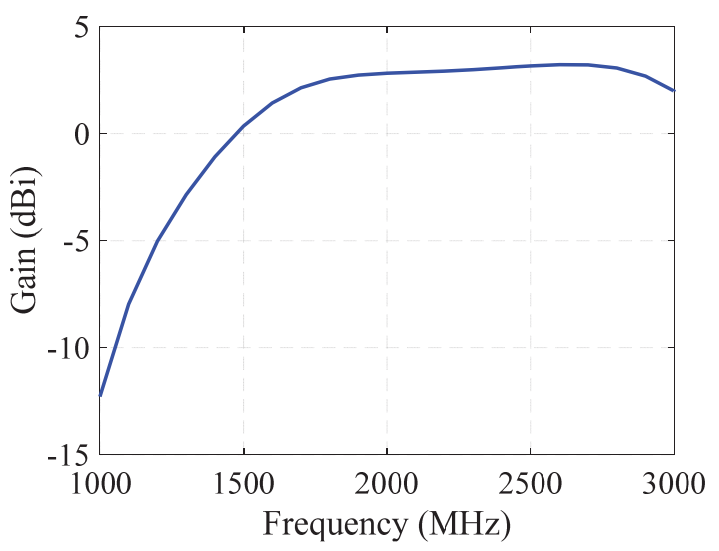

Fig. 6. Antenna gain

The radiation pattern of the antenna presents in figure 7 . The radiation pattern is shown in both $\mathrm{xz}-$ and yz-planes. The proposed antenna is ommi-directional like radiation pattern having a symmetrical pattern with the half-power beam width of 77.8 degree.

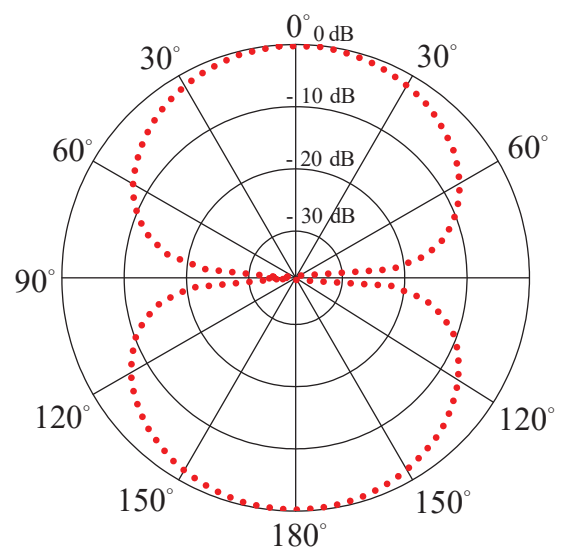

(a) yz-plane

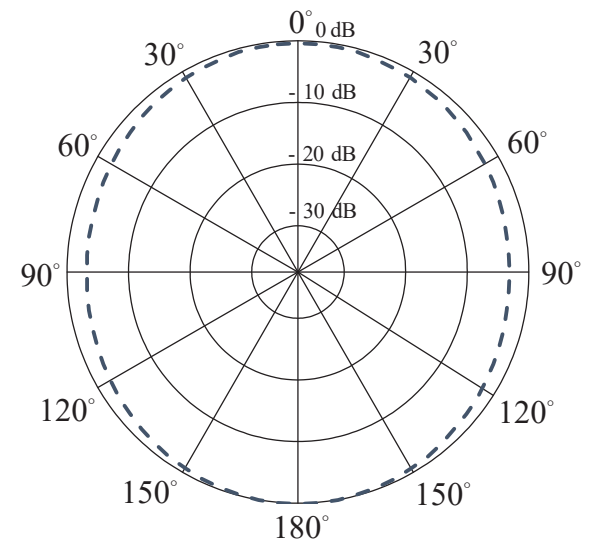

(b) xz-plane

Fig. 7. 2D-Radiation pattern

\section{Conclusion}

The bio-degradable material namely PBS is used to be an antenna substrate. The proposed operating frequency band is from $2400 \mathrm{MHz}$ to $2500 \mathrm{MHz}$. The forming process for PBS substrate is a compression process. The substrate has the thickness of $1 \mathrm{~mm}$ with the dielectric constant of 3.2. The antenna size is $50 \mathrm{~mm} \times 50 \mathrm{~mm}$.

The impedance of the antenna is around $49.2 \mathrm{ohms}$. The impedance bandwidth covers the frequency from 1780 to 2800 which is covered the desired operating frequency. The radiation pattern of the antenna is omnidirectional like with the gain of $3.12 \mathrm{dBi}$. From the results, the PBS-based antenna can be a good candidate for wireless communication system and the PBS is efficient enough to be a material for microwave device in the part of the antenna design.

\section{References}

1. G. Kiemel, IEEE Electromagn. Compat 1, 4, (2012)

2. H-Y.Kang, J.M.Schoenung, Resour Conserv Recycl 45, 4 (2005)

3. L. M. Hilty, Environ Impact Asses 25, 5 (2005)

4. F. F. Rochman, W. S. Ashton, M. G.M. Wiharjo, Dev 24, 1-8(2017)

5. C. A. Balanis, Antenna Theory - Analysis and. Design (John Wileys \& Sons, Inc., 2005)

6. P. Ducheyne, Comprehensive Biomaterials II (Elsevier, Oxford, 2017)

7. M.I. Vladu, Chem Soc Rev 43, 2, 2014 